\title{
Fetal growth and infantile colic
}

\author{
Charlotte Søndergaard, Elisabeth Skajaa, Tine Brink Henriksen
}

\begin{abstract}
Aim-To describe how fetal growth and gestational age affect infantile colic, while considering other potential risk factors. Study design-A population based follow up study of 2035 healthy singleton infants without any disability born to Danish mothers. Information was collected by self administered questionnaires at 16 and 30 weeks of gestation, at delivery, and 8 months post partum. Infantile colic is defined according to Wessel's criteria, but symptoms are restricted to crying for more than three hours a day, for more than three days a week, and for more than three weeks.

Results-The cumulated incidence of infantile colic was $\mathbf{1 0 . 9 \%}$. Low birth weight babies ( $<2500 \mathrm{~g})$ had more than twice the risk (odds ratio $=2.7,95 \%$ confidence interval 1.2 to 6.1 ) of infantile colic when controlled for gestational age, maternal height, and smoking.

Conclusion-Low birth weight may be associated with infantile colic, and further research will be aimed to focus on fetal growth and infantile colic.
\end{abstract}

(Arch Dis Child Fetal Neonatal Ed 2000;83:F44-F47)

Keywords: infantile colic; fetal growth; low birth weight; gestational age

The Danish

Epidemiologic Science Centre, Department of Epidemiology and

Social Medicine,

University of Aarhus,

Denmark

C Søndergaard

The Medical Research

Unit, Ringkjøbing

County, Denmark

C Søndergaard

Perinatal

Epidemiological

Research Unit

Department of

Obstetrics and

Gynaecology, Aarhus

University Hospital,

Denmark

E Skajaa

T B Henriksen

Department of Pediatrics, Aarhus

University Hospital

T B Henriksen

Correspondence to:

Dr Søndergaard, The Danish

Epidemiologic Science

Centre, University Of

Aarhus, Vennelyst Boulevard

6, 8000 Aarhus, Denmark

email: cs@soci.au.dk

Accepted 7 January 2000
Infantile colic is a common condition affecting both parents and the primary health care system. The family often finds it difficult to cope with the infant's excessive crying or fussing, which has been stated as a reason for violence towards the infant. ${ }^{12}$ The prevalence of infantile colic varies from $8 \%$ to $40 \% .^{3}{ }^{4}$ This variation may partly be explained by differences in study design and in the definition of infantile colic. Infantile colic is defined by Wessel $^{5}$ as paroxysms of irritability, crying, or fussing lasting for more than three hours a day, for more than three days a week, and for more than three weeks. It is a self limiting condition most often starting in the first month of life and ending when the infant is $3-4$ months old. Sometimes the symptoms are followed by motor activity, legs drawn up towards the abdomen, distended abdomen, or excessive flatus, but the infants are otherwise healthy and in good condition. ${ }^{5}$

No effective and safe treatment is known, even though the infant may benefit from reduced stimulation or a trial of hypoallergenic formula milk. ${ }^{6}$

The long term consequences of colic are sparsely studied, and the results on child temperament and development are contradictory. ${ }^{7-9} \mathrm{~A}$ small group of infants with early excessive crying may develop a more "persistent mother-infant distress syndrome". ${ }^{10}$

Infantile colic is well described, but its aetiology remains unknown, and many somatic and psychosocial risk factors have been suggested. ${ }^{11-17}$ Many studies have examined possible causes: complications during labour, type of feeding and formula, gastrointestinal motility, parent smoking, and psychological factors before and after delivery at the time of onset of symptoms. However, other prenatal factors may also be of importance. A Swedish population based follow up study has shown a higher s-motilin level in infants who later developed infantile colic. S-motilin was increased in cord blood on the first day, and six weeks and 12 weeks post partum, which indicates gastrointestinal involvement before colic appeared. ${ }^{13}$ It has been hypothesised that depletion of nutrition during critical stages of organ development influences organ functioning, and impaired fetal growth has been associated with a large number of diseases. ${ }^{18}$ Previous studies on birth weight and infantile colic show diverging results, but most have failed to show any association. ${ }^{919-22}$ These divergences may generally be explained by the lack of adjustment for potential confounders and the possibility of recall bias in studies with retrospective data collection and differences in study design. The dual aim of this follow up study is first to describe the association between infantile colic and fetal growth and gestational age, and second to consider other potential risk factors.

\section{Population and methods}

All infants delivered at the Aarhus University Hospital in the period May 1991 to February 1992 and still living in the municipality of Aarhus at the age of 8 months were eligible for the study. Twins, infants with a known handicap, infants with an Apgar score below 7, and infants of non-native mothers were excluded. Information on colic was missing for $4 \%$, which left a total of 1955 infants for analysis.

Information on medical and obstetrical history, smoking, alcohol and caffeine intake, and psychosocial factors during pregnancy was collected by self administered questionnaires at 16 and 30 weeks of gestation. Information about the course of delivery and the newborn was provided by the tending midwife using a structured coding sheet. If the infant was without known handicap or chronic illness, data at 8 months corrected age were collected by a self administered questionnaire completed by the parents and a questionnaire completed by the health visitors at the routine visit, which included an examination of the infant. At this time, information about infantile colic was 
Table 1 The number of children with colic (n), and the cumulated incidence proportion of infantile colic (CIP) according to possible perinatal determinants among 1955 children

\begin{tabular}{lrrr}
\hline & $N$ & $n$ & $C I P(\%)$ \\
\hline Gestational age (completed weeks) & 79 & 13 & 16.5 \\
$\quad<37$ & 1646 & 184 & 11.2 \\
$37-41$ & 227 & 17 & 7.5 \\
$42+$ & 3 & 0 & 0 \\
$\quad$ Missing & 61 & 15 & 24.6 \\
Birth weight (g) & 845 & 91 & 10.8 \\
$\quad<2500$ & 992 & 100 & 10.1 \\
$2500-3499$ & 57 & 8 & 14.0 \\
$3500-4499$ & 1722 & 190 & 11.0 \\
$4500+$ & 230 & 24 & 10.4 \\
Small for gestational age* & 3 & 0 & 0 \\
$\quad$ No & & & \\
Yes & 476 & 56 & 11.8 \\
Missing & 974 & 103 & 10.6 \\
Ponderal index $\left(\mathrm{kg} / \mathrm{m}^{3}\right)$ & 489 & 53 & 10.8 \\
$\quad<23.5$ & 16 & 2 & 12.5 \\
$23.5-26.7$ & & & \\
$26.8+$ & & & \\
Missing & & & \\
\hline
\end{tabular}

${ }^{\star}$ Small for gestational age $=$ newborns with a birth weight below the sex specific 10 th percentile for each completed gestational week.

recalled and recorded both by the parents and the health visitor.

The parent questionnaire defined colic as several hours of crying and described the associated symptoms: legs drawn up towards the abdomen, distended abdomen, and excessive flatus. To the health visitor, colic was specified as more than three hours of crying a day, for more than three days in a week, for more than three weeks, or more than 1.5 hours of crying a day in six out of seven days. ${ }^{23}$ In this study we used information from the parents $(85.2 \%)$, and, if this was missing, we used information from the health visitors $(14.8 \%)$.

The infants were grouped according to birth weight: < 2500, 2500-3499, 3500-4499, and $\geqslant 4500 \mathrm{~g}$. Gestational age at delivery was estimated by ultrasound scanning in early pregnancy $(78 \%)$ or from the first day of the last menstrual period $(6 \%)$. If these data were missing, information about gestational age was obtained from the structured coding sheet completed by the midwife at delivery (15\%). Gestational age was categorised into preterm (<37 completed weeks), term (37-41 completed weeks), and post-term ( $\geqslant 42$ completed weeks). Ponderal index was calculated as birth weight $(\mathrm{kg})$ divided by length at birth $(\mathrm{m})^{3}$. It was categorised into quartiles. Small for gestational age was defined as a birth weight below the 10 th percentile for gestational age in a reference population. The mothers' smoking habits were categorised as non-smoking, smoking one to nine cigarettes a day, and smoking 10 cigarettes or more a day. In this cohort, it has previously been shown that smoking habits remain essentially unchanged from the 16th gestational week throughout pregnancy and during the first eight months post partum. ${ }^{24}$ To obtain as much information as possible on this variable, we therefore used information about smoking at 16 or 30 weeks of gestation $(77.5 \%)$, and, if such information was missing, we used the data from the questionnaire at eight months post partum (16.8\%).

We performed bivariate analyses, stratified analyses, and logistic regression analyses. The logistic regression analyses were performed to control for potential confounding factors. The association between birth weight or gestational age and infantile colic is expressed as an odds ratio (OR) with $95 \%$ confidence interval (CI). A two sided $p$ value of less than 0.05 was considered statistically significant. Factors with a biologically plausible influence on infantile colic were considered potential confounders. The following variables were examined: gestational age, sex, maternal age, maternal height, weight before pregnancy, maternal psychological distress during pregnancy, ${ }^{25}$ cohabitation, educational level, and maternal smoking habits. These variables were entered into the logistic regression as a number of dummy variables equal to the number of categories minus one. The "change-in-estimate" method caused the variable to remain in the final logistic regression model if the estimated risk of infantile colic changed by more than $10 \% .^{26}$

\section{Results}

Of 1955 infants, 214 were reported by their mother or their health visitor to have had infantile colic $(10.9 \%)$. Table 1 shows the cumulated incidence of infantile colic according to birth weight, gestational age, small for gestational age, and ponderal index. Birth weight, gestational age, and smoking were associated with infantile colic in the bivariate analyses. Of the sociodemographic factors considered, older women were at higher risk of having infants with colic than women aged 25-34. Education, years in school, type of residence, and cohabitation were not associated with infantile colic (data not shown).

After adjustment for gestational age, maternal height, and maternal smoking habits, low birth weight babies had more than twice the risk of being reported as having colic than babies with a birth weight between 3500 and $4499 \mathrm{~g}(\mathrm{OR}=2.7,95 \% \mathrm{CI} 1.2$ to 6.1$)$. Table 2

Table 2 Unadjusted (OR) and adjusted odds ratios (aOR) with 95\% confidence intervals for infantile colic

\begin{tabular}{|c|c|c|c|c|c|c|}
\hline & \multicolumn{3}{|c|}{ All children } & \multicolumn{3}{|c|}{ Children $\geqslant 37$ completed gestational weeks } \\
\hline & $N$ & $O R$ & $a O R$ & $N$ & $O R$ & $a O R$ \\
\hline \multicolumn{7}{|c|}{ Birth weight $(\mathrm{g})^{\star}$} \\
\hline$<2500$ & 61 & $2.9(1.6-5.4)$ & $2.7(1.2-6.1)$ & 24 & $3.7(1.5-8.5)$ & $3.3(1.3-8.5)$ \\
\hline $2500-3499$ & 845 & $1.1(0.8-1.5)$ & $1.0(0.7-1.4)$ & 808 & $1.1(0.8-1.5)$ & $1.0(0.7-1.4)$ \\
\hline $3500-4499$ & 992 & 1 (reference) & 1 (reference) & 987 & 1 (reference) & 1 (reference) \\
\hline $4500+$ & 57 & $1.5(0.7-3.2)$ & $1.5(0.7-3.4)$ & 57 & $1.5(0.7-3.2)$ & $1.5(0.7-3.4)$ \\
\hline \multicolumn{7}{|c|}{ Gestational age (completed weeks) $\dagger$} \\
\hline$<37$ & 79 & $1.6(0.8-2.9)$ & $0.9(0.4-2.0)$ & & & \\
\hline $37-41$ & 1646 & 1 (reference) & 1 (reference) & 1646 & 1 (reference) & 1 (reference) \\
\hline $42+$ & 227 & $0.6(0.4-1.1)$ & $0.6(0.4-1.1)$ & 227 & $0.7(0.4-1.1)$ & $0.6(0.4-1.1)$ \\
\hline
\end{tabular}

*Adjusted for gestational age, maternal height, and smoking.

†Adjusted for birth weight, maternal height, and smoking. 
Table 3 Association between infantile colic and small for gestational age expressed by unadjusted (OR) and adjusted odds ratio (aOR) with $95 \%$ confidence intervals among 1952 children

\begin{tabular}{lcll}
\hline & OR & $a O R$ \\
\hline \multicolumn{2}{l}{ Small for gestational age } & & \\
No & 1722 & 1 (reference) & 1 (reference) \\
Yes & 230 & $0.9(0.6-1.5)$ & $0.9(0.6-1.5)$ \\
\hline
\end{tabular}

Table 4 Association between infantile colic and the ponderal index expressed as unadjusted (OR) and adjusted odds ratios (aOR) with 95\% confidence intervals among 1939 infants

\begin{tabular}{llll}
\hline & $N$ & $O R$ & $a O R$ \\
\hline $\begin{array}{l}\text { Ponderal index } \\
\quad<23.5\end{array}$ & 476 & $1.1(0.8-1.6)$ & $1.2(0.8-1.6)$ \\
$23.5-26.7$ & 974 & 1 (reference) & 1 (reference) \\
$26.7+$ & 489 & $1.0(0.7-1.5)$ & $0.9(0.7-1.4)$ \\
\hline * & & &
\end{tabular}

^Adjusted for maternal smoking habits and maternal height.

shows that exclusion of preterm babies failed to change the association (OR $=3.3,95 \%$ CI 1.3 to 8.5). Tables 3 and 4 show the results of logistic regression analyses for small for gestational age and ponderal index. No association was found between small for gestational age or ponderal index and infantile colic.

\section{Discussion}

Infantile colic is a common condition that may be related to low birth weight. We report a risk of infantile colic in infants with a low birth weight that is twice as high as among infants of 2500-3499 g, but we found no association between infantile colic and small for gestational age or ponderal index a birth. This discrepancy could be explained by a higher prevalence of colic in genetically small children than in undernourished children. We tried to differentiate between genetically small children and undernourished children by taking into account maternal birth weight in the analyses on birth weight and infantile colic. Missing data on maternal birth weight, however, of about $30 \%$ limited the validity of this adjustment, and it was difficult to conclude whether the results could be explained by genetic factors or undernourishment.

Only a few previous studies have focused on intrauterine growth, measured as birth weight corrected for gestational age, and infantile colic. Most previous studies have been univariate. Moreover, most of these studies have excluded low birth weight babies or preterm babies, and they have not reported any association between birth weight or gestational age and infantile colic. ${ }^{19}{ }^{27}{ }^{28}$ However, in a follow up study of 249 infants, which included lead exposure and child development, infants with colic were born one week early, and their unadjusted mean birth weight was $200 \mathrm{~g}$ below that of the control group. This follow up study reported an incidence of infantile colic of $17.1 \%$ compared with $10.9 \%$ in our study, probably because their definition of colic was more liberal than ours. They defined colic as crying for more than one hour a day, for an average of at least three days a week, for more than one month. ${ }^{9}$ Another study including
2773 children consulting a paediatrician showed that mean birth weight was lower in the group with colic than the group without (2226 v $3307 \mathrm{~g}) .^{22}$ A study including 67127 babies with birth weights between 2346 and $4312 \mathrm{~g}$ showed a higher birth weight in children with colic than in children without. This study did not take gestational age into consideration. Its first monthly cumulated incidence of infantile colic was $18.3 \% .{ }^{21}$ However, information on colic was based on a simple question to the mother of whether the baby had colic.

The onset of colic is apparently related to time since conception rather than time since birth. The crying peak of preterm babies is at a later age than that of infants born at term. ${ }^{29} \mathrm{We}$ collected data when the infant was about 8 months old to minimise this type of error. One could argue that low birth weight babies are not comparable with the other babies in the cohort because they are often admitted to neonatal care, not only because of severe illness but also because of physiological conditions (feeding difficulties, transient respiratory problems, and icterus). Increased maternal anxiety and separation from the mother may influence the relation between the mother and child as well as the reporting of problems. ${ }^{30}$ This type of bias may also be present in our study.

The diverging results in studies of infantile colic may be explained by the lack of adjustment for potential confounders, the possibility of recall bias in studies with retrospective data collection, and the wide variation in the definition of infantile colic. We have strived to avoid misclassification by seeking information about infantile colic from two different sources, parents and health visitors, neither of whom knew the purpose of the study. The interobserver agreement between the mother's and the health visitors' reporting of colic was good, with a $\kappa$ coefficient of $0.7 .^{31}$ Information about infantile colic was collected up to five months after the symptoms disappeared, which does increase the risk of recall bias, but we doubt that this information is related to birth weight. The parents' information on infantile colic may, however, depend on the infant's actual behaviour at time of recall. The questions on infantile colic included a definition restricted to crying. This has evidently narrowed the definition of colic to the more severe cases, and an incidence of about $11 \%$ is in accordance with other studies using a similar definition. ${ }^{32}$

Missing data on some of the variables may have introduced a bias. We calculated the cumulated incidence of infantile colic in the group with missing data on the variables included in the logistic regression to see if there were any major differences between those with missing values and values not missing. Comparison of the group who did answer the question on infantile colic with the group who did not answer the question showed no differences in maternal age, height, or weight, nor did we find any differences in mean birth weight or gestational age of the infants. Three sets of logistic regression analyses were performed: one including missing values as a separate 
category for each variable; another in which infants with missing values were excluded; and a third in which we looked at birth weight and infantile colic in infants for whom data on maternal smoking and height were missing. We found the same associations between low birth weight and infantile colic in these analyses, but, as expected, confidence intervals were wider when those with missing values were excluded. This study would seem not to be flawed by the traditional selection bias that springs from absence of certain data. However, even if all known potential confounders were duly considered, residual confounding may still exist. Future research will be aimed to focus on fetal growth factors and infantile colic in follow up studies in which prenatal data are collected to minimise the risk of confounding.

We are indebted to Professor Niels Jørgen Secher and Dr Morten Hedegaard for their key roles in initiation of the prenatal cohort, and to the health visitors of the municipality of Aarhus for data collection. We thank Professor Jørn Olsen fo fruitful discussions and comments during the analyses and preparation of the paper. The activities of The Danish Epidemiology Science Centre are financed by a grant from The Danish National Research Foundation. The project was supported by Grant JN 12-1663-1 from the Danish Medical Research Coun-

1 Singer JI, Rosenberg NM. A fatal case of colic. Pediatr Emerg Care 1992;8:171-2.

2 Showers J, Apolo J, Thomas J, Beavers S. Fatal child abuse: a two-decade review. Pediatr Emerg Care 1985;1:66-70.

3 Brazelton T-B. Crying in infancy. Pediatrics 1962 Apr:579-

4 Nylander G, Matheson I. Breast feeding. Effects of smoking and education. Tidsskrift for Den norske Laegeforening and education.

5 Wessel MA. Paroxysmal fussing in infancy sometimes called colic. Pediatrics 1954;14:421-34

6 Lucassen PL, Assendelft WJ, Gubbels JW, van Eijk JT, van Geldrop WJ, Neven AK. Effectiveness of treatments for infantile colic: systematic review. BMF 1998;316:1563-9.

7 Lehtonen L, Korhonen T, Korvenranta H. Temperament and sleeping patterns in colicky infants during the first year of life. $\mathcal{F}$ Dev Behav Pediatr 1994;15:416-20.

8 Rautava PT, Lehtonen L, Helenius H. Infantile colic: child and family three years later. Pediatrics $1995 ; 96: 43-7$.

9 Sloman J, Bellinger DC, Krentzel CP. Infantile colic and transient development lag in the first year of life. Child Psychiatry Hum Dev 1990;21:25-36.

10 Barr RG. Crying in the first year of life: good news in the midst of distress. Child Care Health Dev 1998;24:425-39.
11 Lothe L, Lindberg T. Cow's milk whey protein elicits symptoms of infantile colic in colicky formula-fed infants: double-blind crossover study. Pediatrics 1989;83:262-6.

12 Lothe L, Lindberg T, Jakobsson I. Macromolecular absorption in infants with infantile colic. Acta Paediatr Scand 1990;79:417-21.

13 Lothe L, Ivarsson SA, Ekman R, Lindberg T. Motilin and infantile colic. A prospective study. Acta Paediatr Scand 1990;79:410-16.

14 Hill DJ, Hudson I, Sheffield L. A low allergen diet is a significant intervention in infantile colic: results of a community based study. F Allergy Clin Immunol 1995;96:886-92.

15 Lehtonen L. Gallbladder hypocontractility in infantile colic. Acta Paediatr 1994;83:1174-7.

16 Rautava PT, Helenius H, Lehtonen L. Psychosocial predisposing factors for infantile colic. BMF 1993;307:600-4

17 Carey WB. Maternal anxiety and infantile colic: is there a relationship? Clin Pediatr (Phila) 1968;7:590-5.

18 Barker DJ. In utero programming of chronic disease. Clin Sci (Colch) 1998;95:115-28.

19 Barr RG, Rotman A, Yaremko J, Leduc D, Francoeur TE. The crying of infants with colic: a controlled empirical The crying of infants with colic: a
description. Pediatrics 1992;90:14-21.

20 Illingworth RS. Three months colic. Arch Dis Child 1954;29:165-74.

21 Crowcroft NS, Strachan D P. The social origin of infantile colic: questionnaire study covering 76747 infants. BM7 1997;314:1325-8.

22 Stagnara J, Blanc JP, Danjou G, Simon-Ghediri MJ, Durr F. [Clinical data on the diagnosis of colic in infants. Survey in 2773 infants aged 15-119 days]. Arch Pediatr 1997;4:959-

23 Skajaa E. Sundhedsprofil af småborns og småbornsfamilier i Aarhus. Dansk Sygeplejeråd, 1994.

24 Wisborg K, Henriksen TB, Hedegaard M, Secher NJ. Smoking habits among Danish pregnant women from 1989 to 1996 in relation to sociodemographic and lifestyle factors. Acta Obstet Gynecol Scand 1998;77:836-40.

25 Hedegaard M, Henriksen TB, Sabroe S, Secher NJ. The relationship between psychological distress during pregnancy and birth weight for gestational age. Acta Obstet Gynecol Scand 1996;75:32-9.

26 Mickey RM, Greenland S. The impact of confounder selection criteria on effect estimation. Am $\mathcal{7}$ Epidemiol 1989;129:125-37.

27 Paradise JL. Maternal and other factors in the etiology of infantile colic. $\mathcal{F} A M A$ 1966;197:123-31.

28 Hogdall CK, Vestermark V, Birch M, Plenov G, Toftager Larsen $\mathrm{K}$. The significance of pregnancy, delivery and postpartum factors for the development of infantile colic. F Perinat Med 1991;19:251-7.

29 Barr RG, Chen S, Hopkins B, Westra T. Crying patterns in preterm infants. Dev Med Child Neurol 1996;38:345-55.

30 Humphry RA, Hock E. Infants with colic: a study of maternal stress and anxiety. Infant Mental Health fournal 1989;10:263-72.

31 Christensen JH, Sorensen HT. [Reproducibility of urine strip reactions in medical patients.] Ugeskr Laeger 1989;151:2972-3.

32 Lehtonen L, Korvenranta H. Infantile colic. Seasonal incidence and crying profiles. Arch Pediatr Adolesc Med 1995;149:533-6. 